\title{
Detection of Point Scatterers in Medical Ultrasound
}

\author{
Stine Hverven Thon, Student Member, IEEE, Roy E. Hansen, Senior Member, IEEE, Andreas Austeng, Senior \\ Member, IEEE
}

\begin{abstract}
We present an overview of the detection of point scatterers in ultrasound images and suggest strategies for evaluating and measuring the detection performance. We use synthetic aperture Field II simulations of a point scatterer in speckle background and evaluate how common imaging techniques affect point target detectability. We discuss how to compare different methods and calculate confidence intervals. In general, applying speckle reduction methods reduces the point detection performance. However, the results show that it is possible to smooth the speckle background and preserve relatively high performance with a suitable and optimized method. The different detection performances of the advanced beamforming methods Coherence Factor (CF), Phase Coherence Factor (PCF), and Capon's Minimum Variance (MV) are presented and benchmarked with standard Delay-and-Sum (DAS). The results show that CF achieves slightly better detection performance than DAS for weak point scatterers, whereas PCF and MV perform worse than DAS. Choice of apodization window and adaptive aperture size affects the probability of detection. Results show that methods that preserve spatial resolution have better detection performance of point scatterers.
\end{abstract}

Index Terms-advanced beamformers, detection performance, point scatterer, ultrasound.

\section{INTRODUCTION}

Detection of point scatterers in ultrasound images can be challenging due to peaks in the speckle background. Point scatterers are small, highly coherent targets, and there are several applications in medical ultrasound in which their detection is essential. Breast microcalcifications are small, hard calcium deposits in soft tissue that behave as point scatterers in an ultrasound image and can be an early indicator for cancer [1] [5]. Ultrasound can detect kidney stones, but accurate sizing and diagnosis can be difficult due to background clutter [6], [7]. Point tracking, use of contrast microbubbles, and biopsy needle tracking are other examples where detection of point targets is crucial [8]-[10]. Point scatterers are also of interest in other fields, such as radar [11] and sonar [12].

Detection of a point scatterer in speckle can be viewed as a classical binary detection problem [12]-[16]. In 1983, Smith et al. [17] developed a statistical model for image quality by connecting contrast and lesion detection. Lesion detectability using contrast is now well established in medical ultrasound [18]-[23]. However, the statistical properties of a lesion area are different from a point scatterer. In 1997, Anderson et al. [1] studied the effects of aperture geometry and phase aberration on point detection performance. In [2], they conclude that frequency and spatial compounding slightly improve point

S. H. Thon and A. Austeng are with the Department of Informatics, University of Oslo, P.O.Box 1080, Blindern, NO-0316 Oslo, Norway.

R. E. Hansen is with the Department of Informatics, University of Oslo, Norway, and also with the Norwegian Defence Research Establishment (FFI), P.O.Box 25, NO-2027 Kjeller, Norway. target detectability. They summarize their findings on the effect of phase aberration, sound speed errors, array aperture size, transducer center frequency, speckle reduction by compounding, and logarithmic compression in [3]. In [24], Ouyang et al. reviewed many ultrasound methods for microcalcification detection. Huang et al. [25] created a detection algorithm based on coherence factor. Torp et al. [26] applied detection theory to create a beamformer (BF) for signal detection.

In this paper, we present an overview and framework for the detection of point scatterers in ultrasound images. The motivation for this paper is to present strategies for calculating the detection performance for point scatterers in speckle, how to compare detection performance between different methods, and how common techniques in ultrasound affect the point detection performance. We discuss how to measure the detection performance and calculate confidence intervals. We establish an optimal intensity threshold detector based on standard Delay-And-Sum (DAS) beamforming. Using synthetic aperture Field II simulations [27]-[29], we create images with a point scatterer in uniform speckle background. We vary the applied apodization, aperture size, beamforming, and speckle reduction method. We wish to evaluate how these common ultrasound techniques affect point detection performance. We apply the same detection strategy to the different images and measure the overall detection performances.

Applying an apodization window on the received channel echoes is a common approach to reduce sidelobes at the cost of spatial resolution [30, p. 178] [31, p. 322]. Changes in aperture geometry are expected to give changes in detection performance [1]. We study aperture size to establish how reduced resolution degrades detection.

Speckle reduction methods are often applied on ultrasound images to improve contrast [30, ch. 8.4.6]. Our results show that such methods reduce point detection performance, but the effect varies depending on the chosen method. A suitable speckle reduction method may retain high performance while still applying some smoothing to the background. However, an increased amount of smoothing degrades both the resolution and the detection performance.

Adaptive BFs are applied to improve image quality, and they affect the point detection performance. Using DAS as a reference, we examine several adaptive BFs presented in literature; Coherence Factor (CF) [32], Phase Coherence Factor (PCF) [33], and Capon's Minimum Variance (MV) [34]. Our results show that $\mathrm{CF}$ achieves slightly better detection performance than DAS for weak point scatterers, whereas the other two perform worse than DAS.

The following chapter presents classical detection theory for point scatterers and strategies for calculating point detection performance. We then introduce the background theory for 
the BFs. Section IV presents the different choices for spatial resolution, apodization, and speckle reduction. Section V shows the results, and in Section VI we discuss the effects on detection performance.

\section{BACKGROUND - DETECTION THEORY}

In this section, we present the background theory for how to measure and evaluate detection performance and introduce general detection theory as presented in [12], [13], [16]. We reduce the case to its simplest form and study the detection of a single point scatterer in uniform speckle background. Speckle is caused by interference in the echoes from many random scatterers within a resolution cell [35, ch. 8.2.1], whereas point scatterers are small and highly coherent targets. The objective is to decide between two hypotheses: speckle background without $\left(\mathcal{H}_{0}\right)$ or with $\left(\mathcal{H}_{1}\right)$ a point signal present.

\section{A. Point Target Detection Strategies}

The probability of false alarm $P_{\mathrm{FA}}$ is estimated on sequences containing only speckle and the probability of detection $P_{\mathrm{D}}$ is estimated on sequences containing one point scatter in speckle. We count the number of intensity values above threshold $\gamma$ in each scenario to find the number of false alarms and true positives. By comparing them to the total number of realizations $R$, we get $P_{\mathrm{FA}}$ and $P_{\mathrm{D}}$. There is a choice in the type of strategy to use when calculating point detection performance. When presented with an ultrasound image, one assumes the strongest scatterer is the most likely point target candidate. However, the measured probability when picking the maximum point target will depend on how many independent pixels we consider. We can check if the chosen location is correct and the point is an actual true positive in a simulated environment. The overall detection performance depends on how we choose to count false alarms and true positives. In this section, we present the theory behind the five possible detection strategies listed in Table I. Based on the results, we choose which strategy to use when measuring the detection performance of the different $2 \mathrm{D}$ ultrasound imaging methods.

Table I: Point Detection Strategies

\begin{tabular}{|c|c|}
\hline Strategy & Description \\
\hline A & $\begin{array}{l}\text { Consider each pixel in the image when counting false alarms } \\
\text { and only known point pixel when counting true positives. }\end{array}$ \\
\hline B & $\begin{array}{l}\text { Consider only the maximum value in the image when counting } \\
\text { both false alarms and true positives. }\end{array}$ \\
\hline $\mathrm{C}$ & $\begin{array}{l}\text { Consider the maximum value within a small image search } \\
\text { window around the known point location when counting both } \\
\text { false alarms and true positives. }\end{array}$ \\
\hline $\mathrm{D}$ & $\begin{array}{l}\text { Consider only the maximum value in the image when counting } \\
\text { false alarms and only known point pixel when counting true } \\
\text { positives. }\end{array}$ \\
\hline $\mathrm{E}$ & $\begin{array}{l}\text { Consider only the maximum value in the image, but addition- } \\
\text { ally check if the chosen maximum is an actual true point when } \\
\text { counting true positives. }\end{array}$ \\
\hline
\end{tabular}

The probability distribution of an ideal point signal in additive white Gaussian noise in complex sequences is statistically equivalent to an ideal point in a critically sampled, fully developed speckle scene. To illustrate the detection theory, we consider a simple one-dimensional (1D) speckle sequence constructed as the complex sum of two normally distributed sequences. Under hypothesis $\mathcal{H}_{1}$, we add a point scatterer with intensity $i_{p}$ at a discrete, random location. We estimate expressions for the detection probabilities of the different strategies using this ideal $1 \mathrm{D}$ case.

As a measure of the point's intensity relative to the speckle background, we calculate the point's SNR metric as

$$
\mathrm{SNR}=10 \log _{10}\left(\frac{i_{p}}{\beta}\right),
$$

where $i_{p}$ is the point scatterer intensity, and $\beta$ is the average speckle intensity. In this ideal $1 \mathrm{D}$ study, $\beta=2$.

\section{B. Probability of False Alarm}

The probability density function (PDF) for speckle in an ultrasound image is Rayleigh distributed in amplitude $a$ [36] $[11$, p. 88] [13, p. 30]

$$
p(a)_{\text {Rayleigh }}= \begin{cases}\frac{a}{\sigma^{2}} \exp \left(-\frac{a^{2}}{2 \sigma^{2}}\right) & \text { if } a>0 \\ 0 & \text { if } a<0\end{cases}
$$

Here $\sigma$ is the scale parameter and $\sigma \sqrt{\pi / 2}$ is the mean value. When searching for a point target, one assumes the point has a higher intensity than the surrounding background. To find candidate points, we apply a threshold on the intensity image [11, ch. 10]. Intensity $i=a^{2}$ has a negative exponential PDF $[12$, p. 261] [11, p. 88] [35, p. 502]

$$
p(i)_{\text {neg.exp. }}=\frac{1}{\beta} \exp \left(-\frac{i}{\beta}\right),
$$

where $\beta$ is the mean intensity value and $\beta=2 \sigma^{2}=2$. The threshold $\gamma$ can be found by integrating the PDF for observation $t$ of hypothesis $\mathcal{H}_{0}$ to the chosen $P_{\mathrm{FA}}$ value [13, p. 30]

$$
P_{\mathrm{FA}}=\int_{\gamma}^{\infty} p\left(t ; \mathcal{H}_{0}\right) d t
$$

Inserting (3) into (4), we get

$$
P_{\mathrm{FA}}(\gamma)=\exp (-\gamma / \beta)
$$

where $\gamma$ is the chosen intensity threshold. When measuring the $P_{\mathrm{FA}}(\gamma)$, we simply count the number of false alarms above the threshold $\gamma$ compared to the total number of realizations.

\section{Probability of False Alarm for Maximum Intensity Value}

Strategies B-E selects the maximum value for false alarm calculation. The maximum value of speckle increases however with the number of independent pixels $N$ considered. This increases or inflates the $P_{\mathrm{FA}}$ for a given threshold, as shown in Fig. 1. Following [15, ch. 2.11], the probability is such that $\operatorname{Pr}\left\{i_{\max } \leq \gamma\right\}=(\operatorname{Pr}\{i \leq \gamma\})^{N}$. The $P_{\mathrm{FA}}$ for the maximum of $N$ random independent variables then becomes [15, ch. 2.11], [13, p. 283], [12, p. 587]

$$
P_{\mathrm{FA}}(\gamma)_{\max }=1-\left(1-P_{\mathrm{FA}}(\gamma)\right)^{N}=1-(1-\exp (-\gamma / \beta))^{N} .
$$




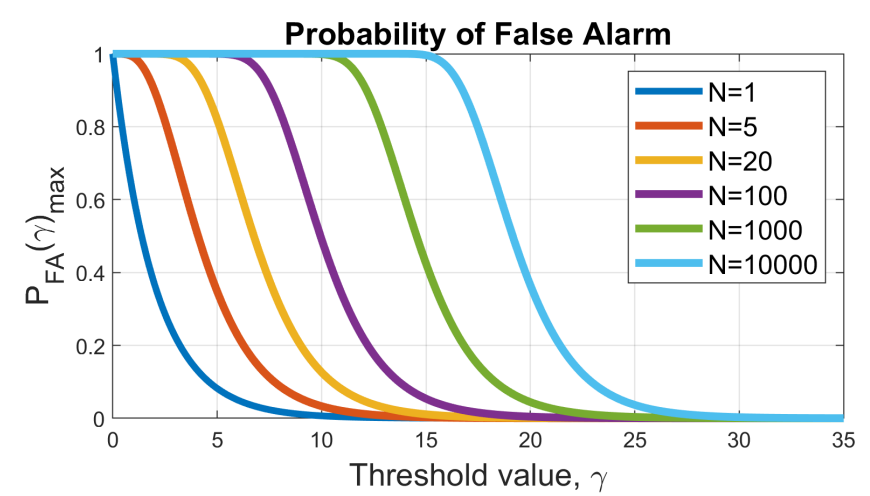

Fig. 1. Theoretical $P_{\mathrm{FA}}(\gamma)_{\max }$ vs. threshold values. The maximum value of a speckle sequence increases with sequence length. The theoretical mean value $\beta$ is 2 .

\section{Probability of Detection}

When a signal from a point scatterer is added to a speckle background, the PDF becomes Rician [13, p. 31], [11, p. 113]

$$
p(a)_{\text {Rician }}= \begin{cases}\frac{a}{\sigma^{2}} \exp \left(-\frac{\left(a^{2}+\alpha^{2}\right)}{2 \sigma^{2}}\right) \mathrm{I}_{0}\left(\frac{\alpha a}{\sigma^{2}}\right) & \text { if } a>0 \\ 0 & \text { if } a<0\end{cases}
$$

where $\mathrm{I}_{0}(u)$ is the modified Bessel function of the first kind and zeroth order, and $\alpha^{2}$ equals the point scatterer intensity $i_{p}$. If $\alpha=0$, the PDF reduces back to Rayleigh. The right-tail probability can be shown to be related to that of the noncentral $\chi^{2}$ random variable and must be evaluated numerically [13, App. 2D] [14, ch. 6.4]. The theoretical $P_{\mathrm{D}}$ for threshold $\gamma$ can be estimated as [13, p. 283]

$$
P_{\mathrm{D}}(\gamma) \approx \mathcal{Q}_{\chi_{2}^{\prime 2}(\tau)}\left(\frac{2 \gamma}{\beta}\right)
$$

where $\mathcal{Q}_{\chi_{2}^{\prime 2}(\tau)}$ is the right-tail probability or complementary cumulative distribution function related to a noncentral $\chi^{2}$ variable. $P_{\mathrm{D}}$ is estimated using $\tau=\frac{2 i_{p}}{\beta}$, and it is therefore dependent on point SNR. By combining (5) with $(8), P_{\mathrm{D}}$ can be expressed in terms of $P_{\mathrm{FA}}$ as

$$
P_{\mathrm{D}}(\gamma) \approx \mathcal{Q}_{\chi_{2}^{\prime 2}(\tau)}\left(2 \ln \frac{1}{P_{\mathrm{FA}}(\gamma)}\right) .
$$

By combining (6) with (8), $P_{\mathrm{D}}$ given by $P_{\mathrm{FA}}(\gamma)_{\max }$ is

$$
P_{\mathrm{D}}(\gamma) \approx \mathcal{Q}_{\chi_{2}^{\prime 2}(\tau)}\left(-2 \ln \left(1-\left(1-P_{\mathrm{FA}}(\gamma)_{\max }\right)^{\frac{1}{N}}\right)\right) .
$$

The $P_{\mathrm{D}}$ of the maximum value can be found as $[12, \mathrm{p} .588]$

$$
P_{\mathrm{D}}(\gamma)_{\max }=1-\left(1-P_{\mathrm{D}}(\gamma)\right)\left(1-P_{\mathrm{FA}}(\gamma)_{\max }\right)^{\left(1-\frac{1}{N}\right)} .
$$

Scalloping loss occurs when signals arrive between two samples [37]. A slight shift in location can cause a reduction in amplitude and energy leakage to the nearby pixels. Oversampling can help reduce this loss and ensure that the maximum achievable resolution is retained [38, ch. 6.7]. The increase in $P_{\mathrm{FA}}(\gamma)_{\max }$ when $N$ increases is less than the alternative loss in signal-to-noise ratio (SNR), and oversampling is therefore beneficial for detection [12, p. 497].

As with $P_{\mathrm{FA}}(\gamma)_{\max }, P_{\mathrm{D}}(\gamma)_{\max }$ also increases with $N$. Fig. 2 shows the different methods for calculating $P_{\mathrm{D}}$. One can consider the known true point location, pick the maximum intensity peak of the whole vector, or pick the maximum within a small search window at the known point position. Since the maximum intensity peak may be in the background for low point SNRs, an option is to additionally check if the found candidate is the true point target. For weak point scatterers, the found candidate may be false, and $P_{\mathrm{D}}(\gamma)$ true max will consequently not converge to 1 for low thresholds.

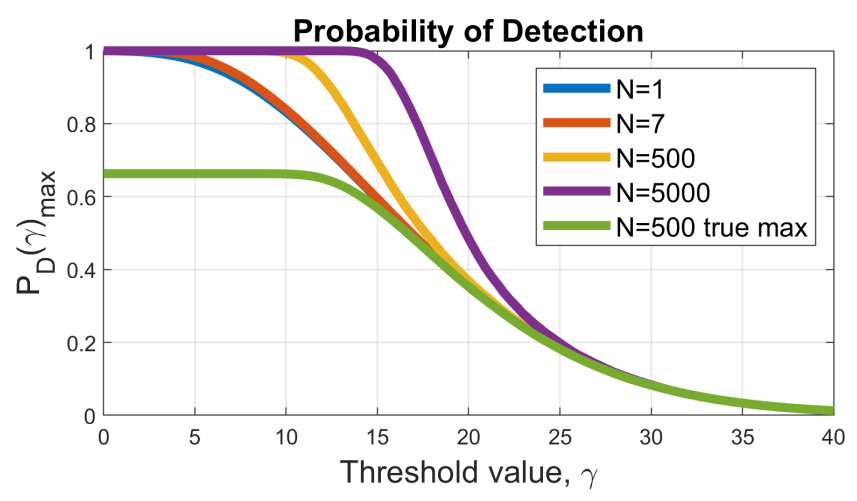

Fig. 2. Theoretical $P_{\mathrm{D}}(\gamma)_{\max }$ vs. threshold values and varying sequence length. Point SNR is $12 \mathrm{~dB}$. The theoretical mean value $\beta$ is $2 . P_{\mathrm{D}}(\gamma)_{\max }$ finds the maximum intensity point within the sequence. $N=1$ signifies $P_{\mathrm{D}}$ calculated using known true point position only, $N=7$ corresponds to a \pm 3 pixel search window, and $N=500$ signifies picking the maximum out of 500 pixels. $P_{\mathrm{D}}(\gamma)_{\text {true max }}$ additionally checks if the found maximum has correct position, and for weak point scatterers it will not converge to 1 .

In Table II, we summarize the theoretical formulae corresponding to the five strategies in Table I. The formulae are for ideal signals in additive white Gaussian noise in complex sequences, which is statistically equivalent to ideal points in fully developed speckle and corresponds to DAS beamforming.

Table II: Summary of Theoretical Formulae for the Strategies

\begin{tabular}{l|l} 
Strategy & Theoretical Formulae for $P_{\mathrm{FA}}$ and $P_{\mathrm{D}}$ \\
\hline A & (5), (8) and (9). \\
B & (6) and (11). \\
C & (6) and (11) with a small number of independent pixels $N$. \\
D & (6), (8) and (10). \\
E & (6). \\
\hline
\end{tabular}

\section{E. Evaluation of Detection Performance}

A Receiver Operating Characteristics (ROC) curve is a standard method of displaying detection performance. It compares $P_{\mathrm{D}}$ to $P_{\mathrm{FA}}$ for a given threshold $\gamma$. By increasing $\gamma$, a lower $P_{\text {FA }}$ can be obtained, but then $P_{\mathrm{D}}$ will also decrease. All points on the ROC curve should satisfy $P_{\mathrm{D}} \geq P_{\mathrm{FA}}[16$, ch. 2.4.2] [13, p. 74]. Fig. 3 presents theoretical ROC curves for the strategies in Table I, applied to a random vector of length 500 and a point scatterer placed at random position with $12 \mathrm{~dB}$ SNR. Strategy $\mathrm{C}$ applied a \pm 3 pixel search window $(N=7$ independent pixels). 


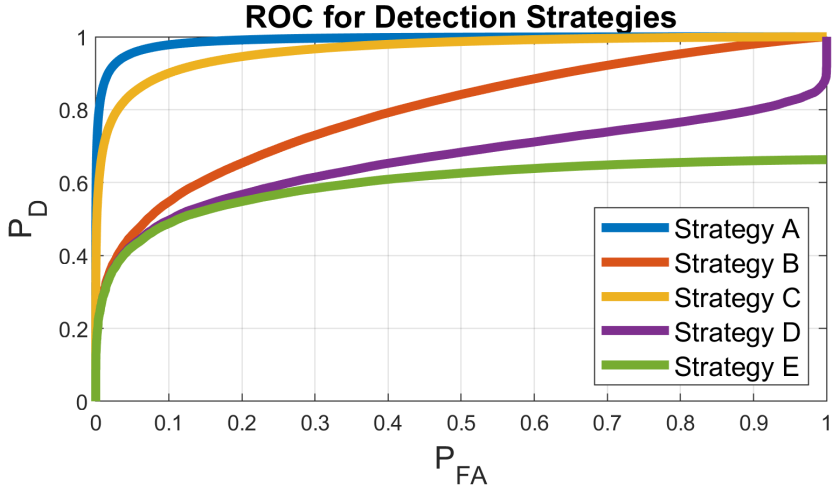

Fig. 3. ROC curves for the different strategies for calculating point detection performance.

Tabulating Area Under the Curve (AUC) is also a way to present ROC results [12, p. 315]. AUC for a diagonal line with $P_{\mathrm{D}}=P_{\mathrm{FA}}$ is 0.5 . Another way to present detection performance is to plot $P_{\mathrm{D}}$ as a function of SNR for a fixed $P_{\mathrm{FA}}$ value $[14$, ch. 7.3.2]. From Fig. 4 we can see that the detection performance of strategy $\mathrm{C}$ varies quite a lot in the range SNR $=[8,14] \mathrm{dB}$. When testing the detection performance of different methods, the SNR-range where $P_{\mathrm{D}}$ varies greatly is the most interesting to analyze. Tabulating $P_{\mathrm{D}}$ for a chosen $P_{\mathrm{FA}}$ value, for example $P_{\mathrm{FA}}=1 \%$, is another way to compare detection performance.

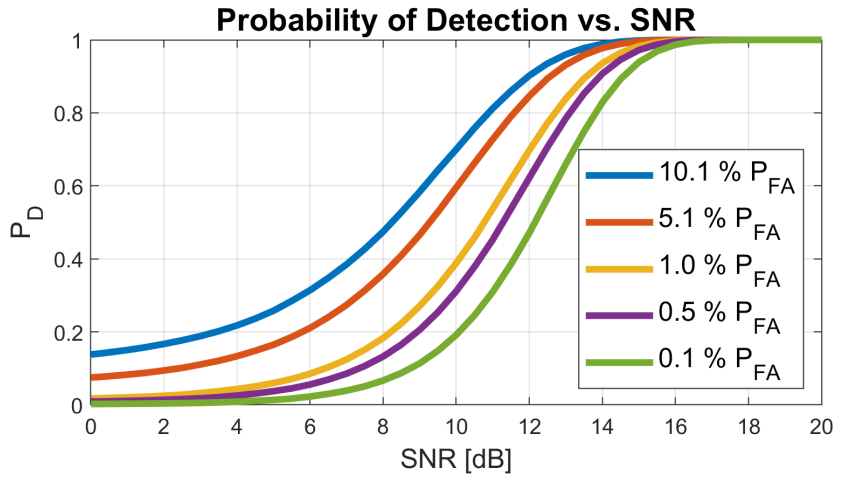

Fig. 4. The $P_{\mathrm{D}}$ increases with point SNR. $P_{\mathrm{D}}$ is shown for five $P_{\text {FA }}$ values and calculated using strategy $\mathrm{C}$ with a \pm 3 pixel search window.

\section{F. Choice of Point Target Detection Strategy}

Strategy A considers only the known point location for detection and can be vulnerable when imaging effects slightly alter the point target's location. Picking the maximum intensity is the natural choice when searching for a point target in speckle background. Strategy A does not pick the maximum value. Strategy B uses maximum for both $P_{\mathrm{FA}}$ and $P_{\mathrm{D}}$, but ignores the fact that the found maximum might not be the true point target. Strategies D and E pick the maximum intensity peak of the entire image for $P_{\mathrm{FA}}$. Two downsides of strategy $\mathrm{D}$ are that we ignore false detections when the maximum is not in the correct position, and only $P_{\mathrm{FA}}$ is inflated by maximum.
Strategy E resembles what we would apply in practice if searching for one point scatterer in an image. However, it has the disadvantage that $P_{\mathrm{D}}$ does not converge to 1 for $P_{\mathrm{FA}}=1$ in the ROC curve if the SNR is low. As such, strategy E does not fulfill the properties of a valid ROC curve [16, ch. 2.4.2].

Strategy C stands out as the optimal choice, a combination of strategies A and B. It resembles a realistic, practical approach the most and gives valid ROC curves. It evaluates a search window around the known point location and picks the maximum value for both false alarm and true positive detection. Fig. 2 shows how $P_{\mathrm{D}}$ for a small search window only slightly deviates from $P_{\mathrm{D}}$ at the known point position. At the same time, evaluating an image selection will give some inflation of $P_{\mathrm{FA}}$ due to the number of pixels considered, as shown in Fig. 1. Picking the maximum gives dependence on $N$ and reduces $R$ compared to using all the pixels.

In the $2 \mathrm{D}$ study, we set the size of the search window to be twice the $-6 \mathrm{~dB}$ spatial resolution for the DAS image. The probability of detecting the true point target as the maximum is high within such a small window, even when scalloping loss or other imaging effects cause a slight shift in pixel location. We also evaluate how the imaging method affects the speckle background and corresponding false alarms by applying a search window. For detection studies with experimental images, we can fit the search window size to the confidence interval of the known point location. Strategy $\mathrm{C}$ can be applied if the detection of several point scatterers is of interest. However, we must note that the choice of search window size relates to the separability of detected point scatterers. If two point targets are located within the same search window, only one detection will be registered. With nonuniform backgrounds, we can apply a form of constant false alarm rate detection with local adaptive thresholding [12], [16]. In our study, we only consider a single point scatterer in homogeneous background speckle.

\section{G. Number of Required Realizations and Confidence Interval}

The number of realizations $R$ will affect the accuracy of the measured performance results. If the true probability is small, for example $P_{\mathrm{FA}}=0.1 \%$, only one in a thousand realizations is expected to exceed the threshold. In such a case, $R$ must be much larger than a thousand to ensure an accurate probability estimate. As presented in [13, p. 37], if we wish to have a relative absolute error $\epsilon$ for probability $P$ for $100(1-b) \%$ of the time, then the required number of realizations $R_{r e q}$ is

$$
R_{r e q} \geq \frac{\left(\mathcal{Q}^{-1}(b / 2)\right)^{2}(1-P)}{\epsilon^{2} P} .
$$

Here $\mathcal{Q}^{-1}(b / 2)$ is the inverse right-tail probability function of a standard normal distribution evaluated at $b / 2$. For very small values such as $P_{\mathrm{FA}}=10^{-3}$, a $10 \%$ relative accuracy for $80 \%$ of the time requires $R=164070$ and it can be difficult or impractical to get enough data. On the other hand, if we wish to analyze a probability of $P=0.05$ for $80 \%$ of the time and have $R=6500$, we get an error of $\epsilon=7 \%$. Confidence intervals for the ROC curve can be plotted by calculating the relative error for both $P_{\mathrm{FA}}$ and $P_{\mathrm{D}}$ at each threshold value using 
(12). The coefficient of variation of the estimated probability $P$, i.e. the ratio of the standard deviation (STD) to the mean of the estimate, is a similar quantity used to express $R_{r e q}[12$, p. 314].

As presented by Hanley and McNeil [39], we can compute the confidence interval for the AUC. For large samples, the distribution of AUC is approximately normal. A $100(1-b) \%$ confidence interval for sample AUC-value $\theta$ may be computed using the standard error (SE) as follows

$$
\theta \pm \mathcal{Q}^{-1}(b / 2) \operatorname{SE}(\theta)
$$

where

$\mathrm{SE}(\theta)=\sqrt{\frac{\theta(1-\theta)+\left(R_{p}-1\right)\left(Q_{1}-\theta^{2}\right)+\left(R_{s}-1\right)\left(Q_{2}-\theta^{2}\right)}{R_{s} R_{p}}}$.

It is worth noting that $\operatorname{SE}(\theta)$ is inversely proportional to $\sqrt{R}$. Quadrupling $R$ only reduces $\operatorname{SE}(\theta)$ by a factor of two. $\operatorname{SE}(\theta)$ is small for high $\theta$ values close to 1 . The number of realizations with and without a point scatterer present is $R_{p}$ and $R_{s}$ respectively. $Q_{1}$ and $Q_{2}$ are distribution-specific quantities expressed as functions of $\theta$ and give a conservative estimate of $\operatorname{SE}(\theta)$ [39].

$$
Q_{1}=\frac{\theta}{2-\theta}, \quad Q_{2}=\frac{2 \theta^{2}}{1+\theta} .
$$

\section{H. Practical Detection Performance in Ultrasound Images}

The theoretical formulae for detection performance in this section are for ideal signals in additive white Gaussian noise in complex sequences. This is statistically equivalent to ideal points in fully developed speckle. In practical ultrasound imaging, several factors that potentially affect detection:

- Additive noise on channel data. The effect of noise causes the SNR to vary with depth.

- Finite probe size causes targets positioned far off-center not to be as well represented as centered point targets.

- The spatial resolution is determined by the aperture size and transmitted pulse bandwidth. It typically varies for depth and angle, and oversampling is common.

- Scalloping loss can cause a reduction in amplitude and leakage in energy to nearby pixels.

- Apodization changes resolution and reduces side lobes.

- Speckle reduction methods are often applied on ultrasound images and alter the statistics.

- Advanced beamforming methods alter both the speckle statistics [40] and the point-plus-speckle statistics.

In the $2 \mathrm{D}$ study, we evaluate how common imaging techniques affect the detection performance of point scatterers.

\section{BACKGROUND - ADVANCED BEAMFORMING}

This section briefly introduces the theory for the following beamforming methods: DAS, MV, CF, and PCF. See The Ultrasound Toolbox (USTB) [41] for implementation.

\section{A. Conventional Delay-And-Sum (DAS)}

Conventional DAS consists of applying a delay and an amplitude weight to the output of each sensor, then summing the resulting signals [31, ch. 4.1]. DAS for image pixel $[z, x]$ is defined as

$$
S_{\text {DAS }}[z, x]=\sum_{m=0}^{M-1} w_{m} y_{m}[z, x],
$$

where $M$ is the number of elements, $y_{m}[z, x]$ is the delayed signal received at element $m$, and $w_{m}$ is a predefined weight. DAS is the oldest and simplest array signal processing algorithm but remains a powerful approach today [31, ch. 4.1].

\section{B. Capon's Minimum Variance (MV)}

Capon's Minimum Variance (MV) [34] calculates for each pixel a data dependent set of weights $w=$ $\left[w_{0}, w_{1}, \ldots, w_{M^{\prime}-1}\right]^{T}$ that minimizes power while maintaining unity gain in the steering direction [42]. To calculate the weights, the spatial covariance matrix needs to be estimated for each pixel. To do this, we apply spatial averaging with subarrays $\boldsymbol{y}_{l}[z, x]=\left[y_{l}, y_{l+1}, \ldots, y_{l+L-1}\right]^{T}, l \in\left[0, M^{\prime}-L-1\right]$, where $M^{\prime}$ is the length of the active receive aperture and $L=M^{\prime} / 2$. We apply time averaging with $1.5 \lambda$ range ( $\lambda$ being the wavelength), a diagonal loading factor of $1 / 100$, and the steering vector as a vector of ones [43]. The MV weights are used in (15) and the final image becomes

$$
S_{M V}[z, x]=\frac{1}{M^{\prime}-L+1} \sum_{l=0}^{M^{\prime}-L} \boldsymbol{w}^{H}[z, x] \boldsymbol{y}_{l}[z, x] .
$$

MV can achieve low sidelobe levels and a narrow beamwidth, increasing the spatial resolution of closely spaced point scatterers. See [43] for a discussion on the parameters.

\section{Mallart-Fink Coherence Factor $(C F)$}

The Coherence Factor ( $\mathrm{CF}$ ) calculates the ratio between coherent and incoherent energy across the aperture [32]

$$
\mathrm{CF}[z, x]=\frac{\left|\sum y_{m}[z, x]\right|^{2}}{M \sum\left|y_{m}[z, x]\right|^{2}} .
$$

It has the potential to give increased contrast and resolution when applied as an adaptive weight to the DAS image [44]

$$
S_{\mathrm{CF}}=S_{\mathrm{DAS}}[z, x] \mathrm{CF} \text {. }
$$

\section{Camacho-Fritsch Phase Coherence Factor (PCF)}

The Phase Coherence Factor (PCF) [33] calculates for each pixel an adaptive weight based on the phase of the receive data. It is a method proposed to improve resolution [45]

$$
\operatorname{PCF}[z, x]=\max \left\{0,1-\frac{\gamma^{*}}{\sigma_{0}} f[z, x]\right\},
$$

where $\gamma^{*}$ is a parameter provided to adjust the sensitivity of PCF to out-of-focus signals [33], and $\sigma_{0}=\pi / \sqrt{3}$ is the STD of a uniform distribution between $-\pi$ and $\pi$ [41]. The function $f[z, x]$ calculates the minimum STD of the instantaneous phase across the aperture. PCF is applied as an adaptive weight to the DAS image

$$
S_{\mathrm{PCF}}=S_{\mathrm{DAS}}[z, x] \mathrm{PCF} .
$$




\section{METHODS}

In this section, we describe the simulation, processing, and test setups in our study. We used Field II to generate raw channel data and USTB to beamform the data, as illustrated in Fig. 5. We varied the following: the simulated phantom, the point data intensity, the aperture size, the apodization, the speckle reduction, and the BF.

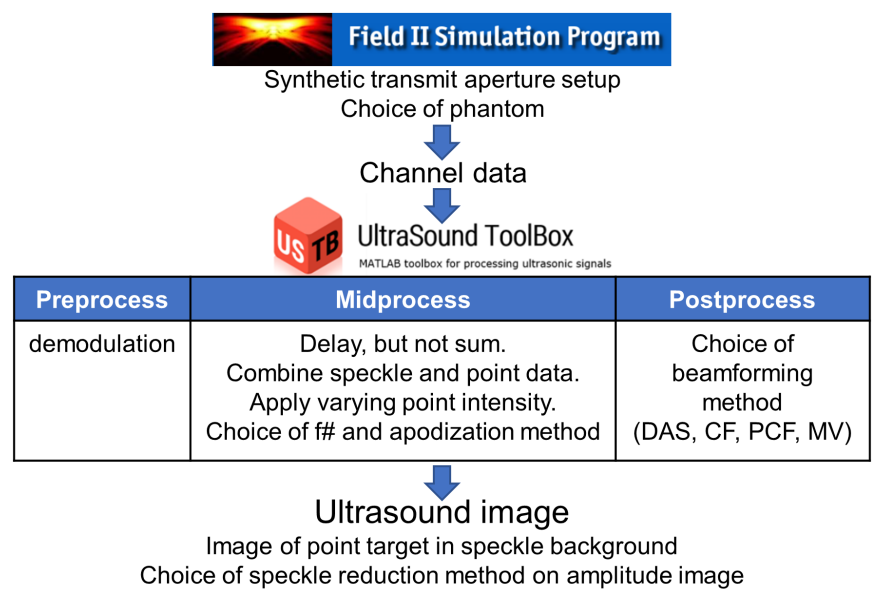

Fig. 5. Overview of the acquisition and processing stage for producing simulated ultrasound images. We use Field II to produce channel data and USTB to beamform the data.

\section{A. Synthetic Transmit Setup and Image Reconstruction}

We designed the phantom as a simple scenario of a single point scatterer in speckle background to establish a baseline detection. We simulated synthetic transmit aperture datasets in Field II to obtain synthetic focus and uniform resolution for all pixels. We used a 128 element, $\lambda$ pitch, linear array with $5.1 \mathrm{MHz}$ center frequency (L11-4v). We added white Gaussian noise to the channel data at $10 \mathrm{~dB}$ channel SNR. We kept the additive channel noise fixed and below the speckle background level and instead varied the point intensity relative to the combined speckle and noise background. The speed of sound in the medium was $1540 \mathrm{~m} / \mathrm{s}$, the transmitted pulse bandwidth was $65 \%$ of the center frequency, the wavelength $\lambda$ was $0.3 \mathrm{~mm}$, and the aperture size was $38.1 \mathrm{~mm}$.

We simulated 200 speckle realizations, each consisting of 91000 point scatterers and at least 20 scatterers per resolution cell. The speckle pattern is considered well developed if the number of scatterers per resolution cell is larger than ten [46] [47, ch. 2.9]. We simulated one point scatterer at 65 image positions $(13 \times 5$ matrix grid), chosen to ensure varying straddle loss. To reduce the number of Field II simulations, we simulated radio frequency channel data for the point scatterers and the speckle background separately. Afterward, we combined the channel data such that we could change the point intensity and position. For a given method and point SNR value, we created and analyzed $65 \times 100$ images. To improve the statistical accuracy at low $P_{\mathrm{FA}}$ values, we additionally analyzed 100 speckle images. With detection strategy $\mathrm{C}$, this corresponds to 6500 realizations for $P_{\mathrm{D}}$ calculation and 13000 realizations for $P_{\mathrm{FA}}$ calculation. To ensure uniform average background intensity, we calculated correction maps from the average of all speckle background realizations for each advanced BF and apodization method and applied them to the images before detection. The beamforming was performed in MATLAB (Mathworks, Natick, MA) using USTB.

The reconstructed image scene was $20 \mathrm{~mm}$ wide and $40 \mathrm{~mm}$ deep. We oversampled the images to ensure full gain and top performance for the high-resolution adaptive BFs. The -6 $\mathrm{dB}$ theoretical and measured spatial resolution for a center point scatterer in DAS with hamming transmit apodization corresponded to approximately $5 \times 7$ pixels. The total image size was $512 \times 256$. For the detection search, we applied a search window grid size two times the spatial resolution. The window ensured we would not miss a detection due to straddle loss or a slight shift in pixel location and also caught some of the method's effect on detected false alarms.

We calculate the point's SNR metric using (1) with the average maximum point intensity and the average intensity of the speckle region around center focus depth without the point scatterer present. SNR was calculated for the point scatterer positioned in the center of the image scene, beamformed by the DAS method. By varying the point's intensity, we tested three SNR values in the range $\mathrm{SNR}=[10,14] \mathrm{dB}$. Detection performance in this range varied greatly and signified relatively weak point scatterers in speckle. Note that the choice of point detection strategy and search area affects which SNR interval to choose.

\section{B. Adaptive Aperture Size and Spatial Resolution}

The ratio between imaging depth $z$ and size of active aperture $D$ is termed $F$-number or $f_{\#}=z / D$. Uniform resolution in the final image can be ensured by having a constant $f_{\#}$. It ensures a range-independent beamwidth by increasing the active aperture with increasing range $z[30, \mathrm{p}$. 381]. For pixels close to the edges, the active aperture will be reduced since the physical aperture has finite size. We used $f_{\#}=1.75$ in this study unless otherwise described. The twoway $-6 \mathrm{~dB}$ lateral resolution with fixed $f_{\#}$ and rectangular apodization can be approximated as [47, p. 12] [37]

$$
x_{\mathrm{res}} \approx \frac{1.21}{\sqrt{2}} \lambda f_{\#} .
$$

We compared three different $f \#$ values to establish how image resolution affects the detection performance.

\section{Apodization}

In medical ultrasound, applying an apodization window is standard practice for reducing sidelobe levels [30, p. 178] [31, p. 322]. Windowing is always a trade-off between resolution and contrast [37]. We applied the following apodization methods to study the effect on detection: rectangular, Hamming only on transmit, and Hamming on both transmit and receive. Excepting the apodization study, we applied the same apodization for all simulations; hamming apodization on transmit and rectangular on receive. 


\section{Speckle Reduction}

Speckle reduction or noise suppression is often applied on medical ultrasound images to improve contrast [30, ch. 8.4.6]. Speckle reduction using filters can greatly improve the contrast between grayscale tissue areas but simultaneously reduce the the point scatterer's resolution. In this study, we analyzed the effect of common denoising filters on the detection of point scatterers. We studied the following filters: Wiener, nonlocal means, bilateral, anisotropic diffusion, and simple block averaging. Table $\mathrm{V}$ in App. A presents the filter settings used in this study. Speckle reduction can be applied on the intensity or log-intensity images, but we applied it on the amplitude DAS image for this study [48]-[50]. Based on visual inspection, we chose filter settings giving a mild background smoothing. For all methods, we found parameter settings giving in approximately 0.3 STD vs. mean $\mu$ of the speckle background. A detailed study on optimal parameters for each filter was not performed.

The Wiener filter is also referred to as the minimum mean square error filter or the least square error filter [51, ch. 5.8]. The method assumes that noise and image are uncorrelated, tailoring itself to the local image variance. We tested four window sizes with an increasing amount of speckle reduction. We also tested four window sizes for simple block averaging.

A non-local means filter removes noise from an image while preserving the sharpness of strong edges [52]. We used the technique first implemented by Buades et al. [52], but for computational efficiency omitted to convolve the Euclidean distance between two comparison windows with a Gaussian kernel. We varied the degree of smoothing with respect to the STD of the image. We present four filters with an increasing amount of speckle reduction.

An anisotropic diffusion filter also tries to denoise an image while still preserving the sharpness of edges. It is a technique presented by Perona and Malik [53]. We tested several filters by varying the number of iterations used in the diffusion process and the gradient threshold value with respect to STD of the image. The threshold value controls the conduction process by classifying gradient values as edges or noise, and increasing the value smooths the image more.

A bilateral filter applies an edge-preserving Gaussian filter. It was presented by Tomasi and Manduchi [54]. We varied the degree of smoothing with respect to the image variance and the STD of the spatial Gaussian smoothing kernel. The value of the degree of smoothing corresponds to the variance of the Range Gaussian kernel of the bilateral filter [54]. The Range Gaussian is applied on the Euclidean distance of a pixel value from the values of its neighbors.

\section{E. Advanced Beamforming Methods}

We analyzed the four BFs presented in Section III and will refer to them as DAS, MV, CF, and PCF. The input to all the methods was identical. We applied Hamming window on transmit and uniform apodization on receive. The point SNR value was calculated from the DAS images.

\section{REsults}

A. Spatial Resolution and Apodization

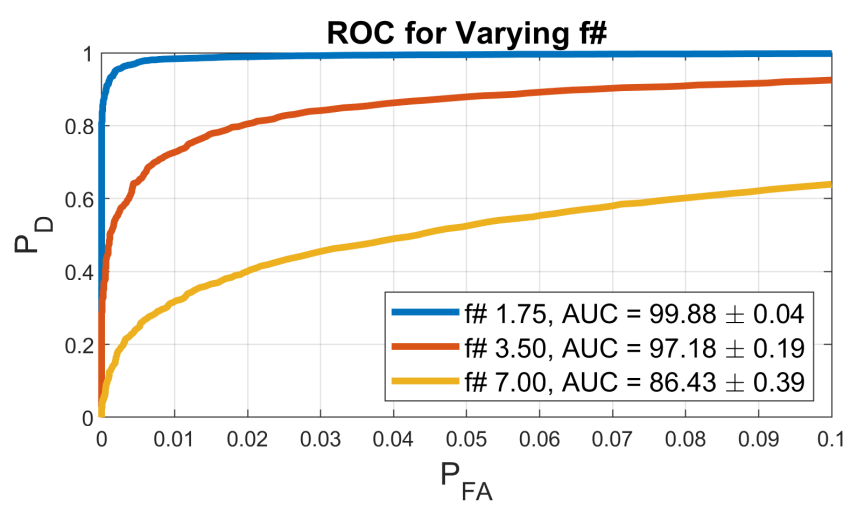

Fig. 6. ROC curve for DAS using three adaptive aperture sizes, giving different spatial resolutions. $P_{\mathrm{FA}}$ is shown up to 0.1.

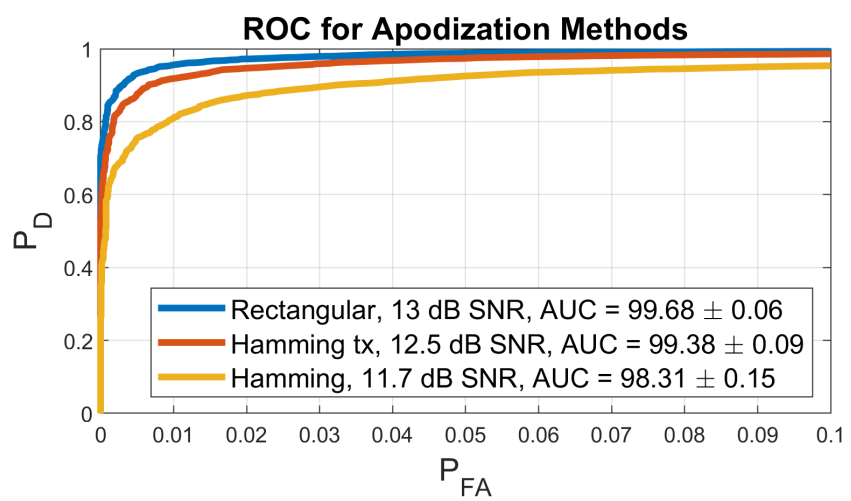

Fig. 7. ROC curve for DAS using different apodization methods: rectangular, Hamming only on transmit (tx), and Hamming on both directions. The input data is the same for all methods, but the different windows alter the spatial resolution and the final point SNR values. $P_{\mathrm{FA}}$ is shown up to 0.1.

Fig. 6 presents the detection performance of three adaptive aperture sizes. As seen from the results, increasing the $f \#$ degrades the detection performance. The performance difference between $f \#=7$ and $f \#=3.5$ illustrates how doubling the adaptive aperture greatly improves point detectability. We applied hamming apodization on transmit and rectangular on receive for all $f \#$ s. Fig. 7 shows the ROC curve for DAS using different apodization schemes: rectangular, Hamming only on transmit, and Hamming on both transmit and receive. Applying a uniform rectangular window gives the highest probability of point detection. A nonuniform window reduces resolution and slightly alters the measured point SNR value. The three methods have final SNR values of 13.0, 12.5, and $11.7 \mathrm{~dB}$.

\section{B. Speckle Reduction}

Fig. 8 shows speckle-reduced images of a sample speckle realization with a point scatterer. Fig. 9 presents AUC results for the methods with respect to STD vs. the mean of the smoothed speckle background. The images in Fig. 8 correspond to the bars with $\sigma / \mu \approx 0.3$ in Fig. 9. Fig. 10 shows 

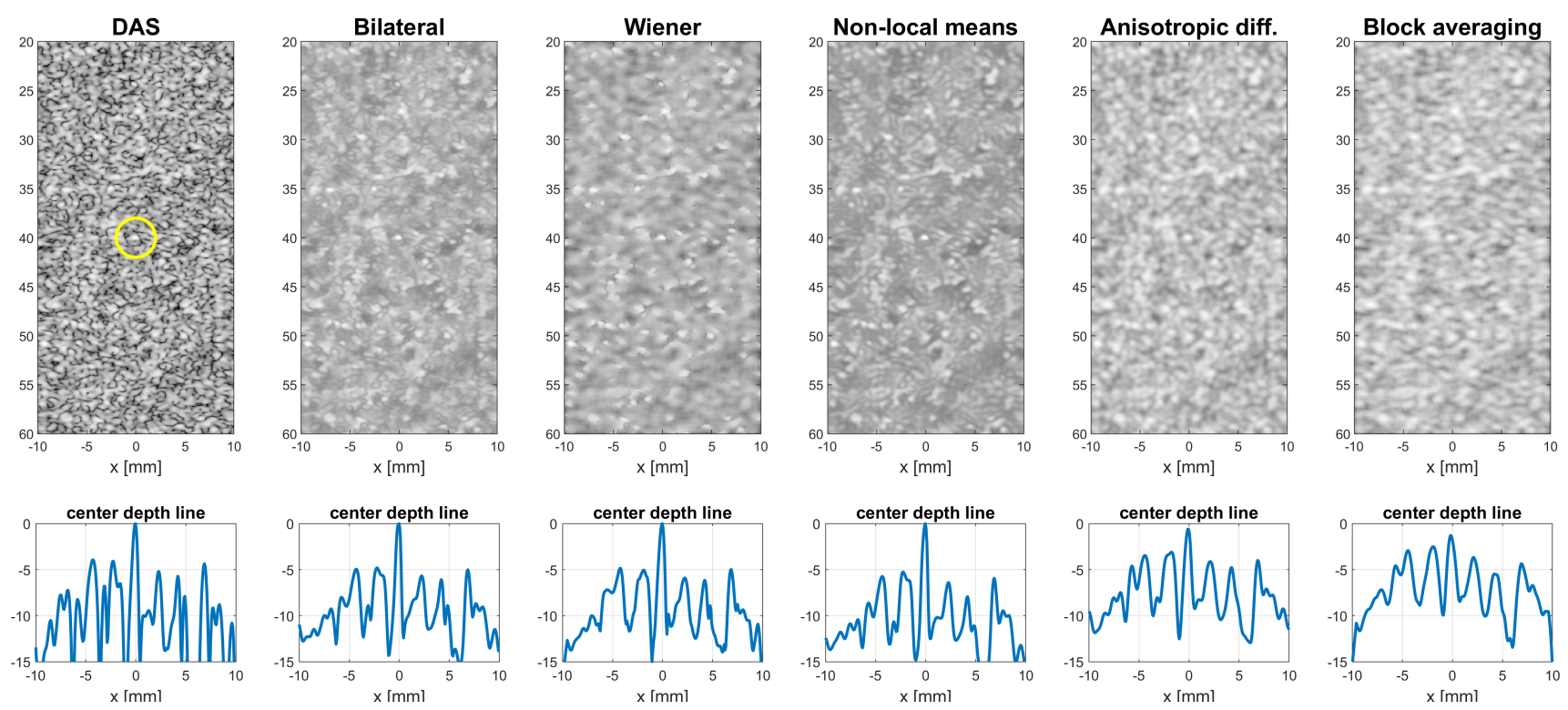

Fig. 8. Speckle reduction methods applied on an image with a point located at $[z, x]=[40,0] \mathrm{mm}$, indicated by the yellow circle. Point SNR is $10.3 \mathrm{~dB}$. All images are normalized by maximum and presented with $-35 \mathrm{~dB}$ dynamic range. A center depth cut is presented below each image. The filters have speckle backgrounds with $\sigma / \mu \approx 0.3$. Thresholding the DAS image at $-3 \mathrm{~dB}$ corresponds to $10 \% P_{\mathrm{FA}}$ and $84 \% P_{\mathrm{D}}$. For this specific DAS image, thresholding at $2 \% P_{\mathrm{FA}}$ gives eight false alarms.

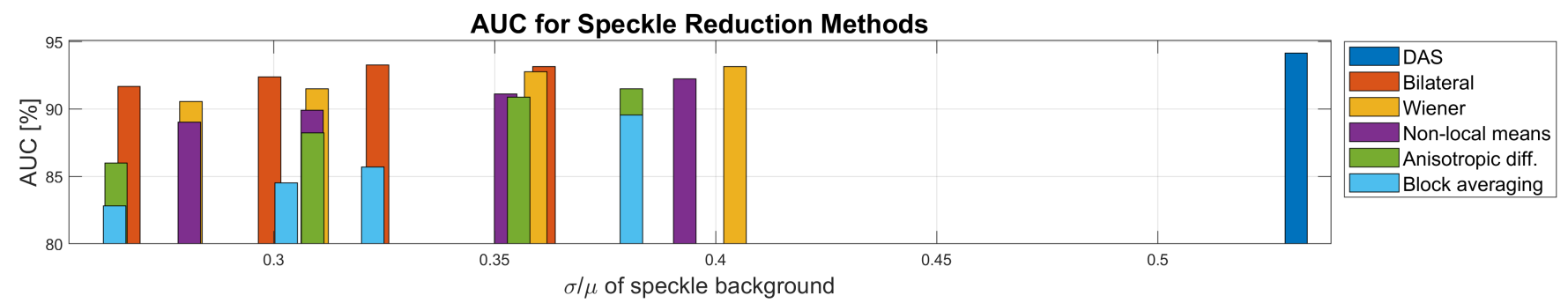

Fig. 9. Tabulated performance results for the speckle reduction methods with $10.3 \mathrm{~dB}$ point SNR. The bars present the filter parameters giving high AUC with respect to STD vs. mean of the speckle background.

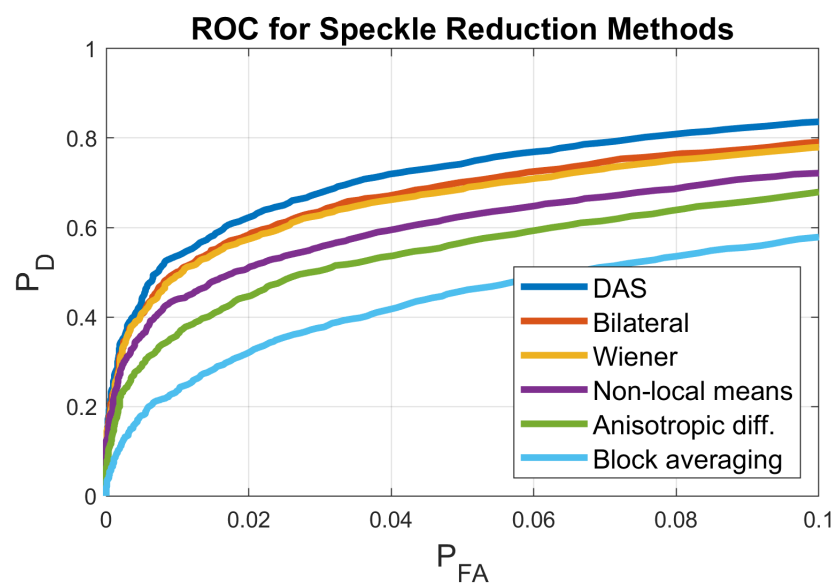

Fig. 10. ROC curves for speckle reduction methods compared to DAS with $10.3 \mathrm{~dB}$ point SNR. $P_{\mathrm{FA}}$ is shown up to 0.1 . The filters give speckle backgrounds with $\sigma / \mu \approx 0.3$. The bilateral and Wiener filter have partially overlapping confidence intervals. the corresponding ROC curves. We can see that some speckle reduction methods perform very close to the original DAS image, and all perform better than simple block averaging. The ROC curves for the bilateral and Wiener filters have partially overlapping confidence intervals. The differences in the ROC curves for the other filters are statistically significant since the separations between the curves are larger than the individual confidence intervals. $80 \%$ confidence intervals were calculated using (12) with 6500 and 13000 realizations for $P_{\mathrm{D}}$ and $P_{\mathrm{FA}}$.

\section{Advanced Beamforming Methods}

Fig. 11 shows beamformed image scenes where the point scatterer is located in the center. The figure visually compares the effect of the BFs. Fig. 12 presents ROC curves for three SNR values. Tabulated performance statistics are shown in Table III and IV. Confidence intervals for the ROC curves were calculated using (12) at each threshold value, with 6500 realizations for $P_{\mathrm{D}}, 13000$ realizations for $P_{\mathrm{FA}}$ and an $80 \%$ confidence interval. Confidence intervals for the tabulated AUC values were calculated similarly using (13). 

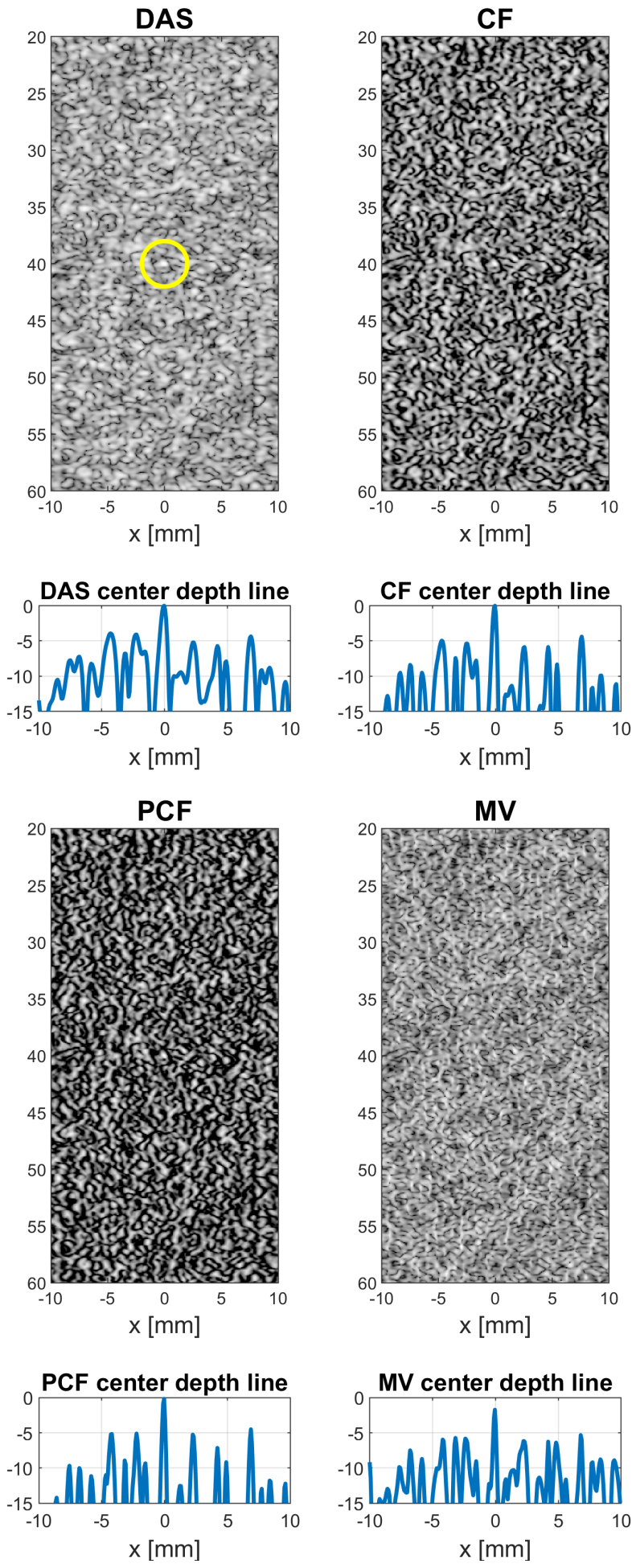

Fig. 11. Four BFs applied on image scene with a point located in center at $x=0 \mathrm{~mm}$ and $z=40 \mathrm{~mm}$ depth. The yellow circle indicates the point location. We analyzed 65 different point locations for each speckle background, resulting in 6500 images per beamforming method per SNR value. Point SNR is $10.3 \mathrm{~dB}$ here. All images are normalized by maximum and presented with a $-35 \mathrm{~dB}$ dynamic range to be comparable for the detectability of the point scatterer. A center depth cut is presented below each image to visualize how much the point stands out in log-intensity from peaks in the surrounding speckle background.

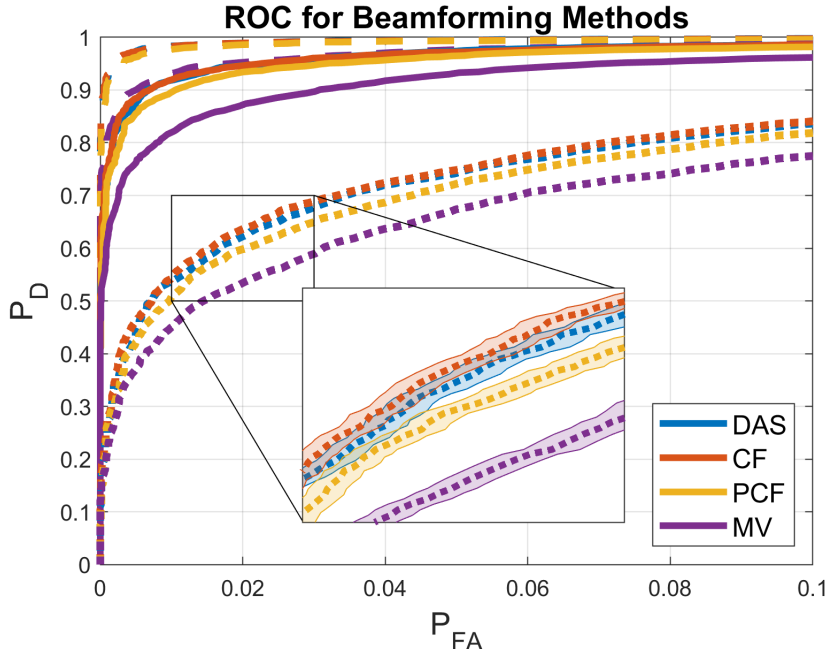

Fig. 12. ROC curves for advanced BFs compared to DAS. Three SNR values are presented; $10.3 \mathrm{~dB}$ (dotted), $12.5 \mathrm{~dB}$ (solid), and $13.7 \mathrm{~dB}$ (dashed). $P_{\mathrm{FA}}$ is shown up to $0.1 .80 \%$ confidence intervals are also shown for a rectangular section of the graph, calculated using 6500 realizations for $P_{\mathrm{D}}$ and 13000 realizations for $P_{\mathrm{FA}}$.

Table III: AUC for Beamforming Methods

\begin{tabular}{llll} 
BF & $10.3 \mathrm{~dB}$ SNR & $12.5 \mathrm{~dB}$ SNR & $13.7 \mathrm{~dB}$ SNR \\
\hline DAS & $94.1 \pm 0.3$ & $99.4 \pm 0.1$ & $99.9 \pm 0.04$ \\
CF & $94.1 \pm 0.3$ & $99.4 \pm 0.1$ & $99.9 \pm 0.04$ \\
PCF & $93.5 \pm 0.3$ & $99.2 \pm 0.1$ & $99.8 \pm 0.05$ \\
MV & $91.9 \pm 0.3$ & $98.4 \pm 0.1$ & $99.4 \pm 0.09$ \\
\hline
\end{tabular}

Table IV: $P_{\mathrm{D}}$ for Beamforming Methods

\begin{tabular}{llll} 
BF & $10.3 \mathrm{~dB}$ SNR & $12.5 \mathrm{~dB}$ SNR & $13.7 \mathrm{~dB}$ SNR \\
\hline DAS & $62.2 \pm 0.8$ & $94.7 \pm 0.4$ & $98.9 \pm 0.2$ \\
CF & $63.6 \pm 0.8$ & $94.6 \pm 0.4$ & $98.9 \pm 0.2$ \\
PCF & $59.8 \pm 0.8$ & $93.4 \pm 0.4$ & $98.6 \pm 0.2$ \\
MV & $53.4 \pm 0.8$ & $87.2 \pm 0.5$ & $95.3 \pm 0.3$ \\
\hline
\end{tabular}

\section{DISCUSSION}

\section{A. Spatial Resolution and Apodization}

Reducing the size of the aperture degrades the spatial resolution of the image. From Section II, we know that reducing the number of independent pixels lowers $P_{\mathrm{FA}}(\gamma)_{\max }$. Increasing the size of the resolution cell will change the point signal to speckle level within the cell. A loss in resolution is accordingly a loss in point SNR. Fig. 6 shows how reducing the aperture size degrades the detection, as also found in [1]. The effect is substantial compared to the other methods in this study. We conclude that it is ideal to have high spatial resolution and high point SNR in terms of detection performance. Techniques that trade-off spatial resolution to obtain better contrast resolution will therefore degrade point detection.

Similarly, we can conclude from the results in Fig. 7 that when we mainly wish to detect point scatterers, the ideal method is to apply uniform apodization. Applying a nonuniform window will suppress some probe elements and thereby reduce the resolution and the maximum point intensity. Apodization with a nonuniform window results in lower point detection performance, though general contrast in the final 
image might have improved. It is also worth noting that point scatterers at the edges can be more challenging to detect since the edges may not have full aperture coverage.

\section{B. Speckle Reduction}

Applying any speckle reduction will smooth the background, but unfortunately also degrade the image resolution [31, p. 322]. This signifies a reduction in point SNR. Based on our results, speckle reduction depletes the number of false alarms but reduces $P_{\mathrm{D}}$ even more. In general, speckle reduction thereby degrades the overall point detection performance.

However, the results also show it is possible to smooth the background and keep detection performance close to the original. In this study, the bilateral, Wiener, and non-local means filters outperformed the others and managed to preserve relatively high detection performance even for a high degree of background smoothing. Optimal parameter choices improve detection performance, but from our experience, it is not likely that this will do better than DAS. The anisotropic diffusion filter did not perform as well as the non-local means and bilateral methods. Block averaging significantly reduced the detection performance and is a poor choice if point targets are of interest. When choosing a method for reducing speckle, one should consider that different methods have different performances concerning point detection.

\section{Advanced Beamforming Methods}

Fig. 12 shows how CF performs slightly better than DAS for low SNR and $P_{\mathrm{FA}}$ values, though the confidence intervals are partially overlapping. Unweighted DAS is the best detector unless prior knowledge is included in the analysis. Point scatterers are small, bright, and highly coherent targets. We can expect high measurable coherence in the signal from a point scatterer, and point detection algorithms can exploit this [25], [55]. CF matches prior knowledge about point targets well since it differentiates between coherent and incoherent energy. Speckle background alone is known [32] to have an expected mean coherence value of $2 / 3$, though this theoretical value is affected by specific circumstances in ultrasound imaging [56]. Since this is the average, the weights of CF will also enhance some pointlike coherent scatterers in the speckle background. It can be difficult to distinguish true point targets from these peaks at low SNRs based on the weights alone. However, since CF uses image weighting, it can achieve slightly better performance than DAS. The difference in detection performance is distinguishable at low SNR and $P_{\mathrm{FA}}$ values. However, we do not have enough realizations to state that the difference is statistically significant since the confidence intervals are partially overlapping in Fig. 12.

The tabulated values for $P_{\mathrm{D}}$ in Table IV show higher values for $\mathrm{CF}$ than DAS with statistical significance with $75 \%$ confidence. The confidence interval curves in Fig. 12 is still a better way to illustrate the accuracy of the results since it shows several measurements. In general, AUC is a more robust method than tabulating $P_{\mathrm{D}}$ for a given $P_{\mathrm{FA}}$ since it integrates several measurements and is thereby less vulnerable to limited amount of realizations. Tabulating AUC will not catch the slight difference between $\mathrm{CF}$ and DAS here since it is calculated over all $P_{\mathrm{FA}}$ values, and the difference is only distinguishable for low $P_{\mathrm{FA}}$ values.

The results indicate that $\mathrm{CF}$ is a promising method to use when point scatterers are of interest, especially in imaging scenes with low SNR values. The DAS image shown in Fig. 11 is an example of such a weak point scatterer. Thresholding the image at $-3 \mathrm{~dB}$ below the maximum intensity retains many false alarms. An example of a practical application is an ultrasound image with small macrocalcifications in early development. The points will then have low SNR values relative to the background. In such a medical scenario, we wish to have few false alarms. A $P_{\mathrm{FA}}$ value of $1 \%$ or lower can be applicable. Referring to the ROC curve in Fig. 12, 1\% $P_{\mathrm{FA}}$ corresponds to detecting half of the weak point scatterers, and the results show that $\mathrm{CF}$ is a promising alternative to DAS.

The three adaptive BFs have different PDFs from DAS [40]. The PDFs of CF and PCF resemble intensity distributions, and in Fig. 11 they appear darker with fewer bright peaks. The difference in PDF affects both $P_{\mathrm{FA}}$ and $P_{\mathrm{D}}$. The difference in ROC for PCF in Fig. 12 is statistically significant since the difference between the other curves is larger than the particular confidence interval. PCF has a statistically significant lower detection performance than $\mathrm{CF}$ and DAS, but the difference in performance decreases with increasing SNR. PCF is calculated from the STD of the phase. A possible explanation for its performance is that PCF disregards amplitude and thereby retains less information about the point target and the speckle background than $\mathrm{CF}$.

In our analysis, MV has lower detection performance than the other methods. The difference is statistically significant for all three SNR values. MV is known to have good spatial resolution and high separability of point targets [57]. MV is designed for optimum performance for signal in interference and noise. We observe more false alarms for MV, as illustrated in Fig. 11 where the true point in the MV image is not the maximum intensity in the image. A possible cause is the strong dependence on the estimate of the spatial covariance matrix. With a true covariance matrix, its performance should meet DAS for our scenario. With parameter choices that have shown to be robust in ultrasound imaging [43], MV enhances more of the speckle background peaks and attains a poorer detection performance. In the case of multiple point scatterers in speckle, we expect MV to distinguish itself in separating weak point scatterers in the presence of other strong point scatterers.

\section{CONCLUSIONS}

In this paper, we have presented an overview of the detection of point scatterers in ultrasound images. Based on classical detection theory, we presented five main strategies to measure and evaluate detection performance. We showed how the choice of detection strategy affects the performance and gives very different ROC curves. We showed how to compare the effect of different imaging methods and calculate confidence intervals for the detection performance.

We simulate many images of a point target in speckle and apply a set of common ultrasound techniques to form 
the images. Our study shows that uniform apodization gives the best performance when detecting a point scatterer in speckle. Applying a Hamming window or similar suppresses information from some probe elements. It reduces the spatial resolution and thereby degrades the detection performance. Similarly, a large aperture will have better spatial resolution and higher detection performance than a smaller one.

In general, applying speckle reduction to an image will reduce the detection performance of point targets. However, our results show that it is possible to smooth the speckle background while still having detection performance quite close to the original, provided one applies a suitable speckle reduction method with optimal parameters.

The advanced BFs PCF and MV have lower point detection performance than DAS. Unweighted DAS is the best detector unless prior knowledge is included in the analysis. However, CF matches prior knowledge about point targets well. Since it differentiates between coherent and incoherent energy across the aperture, its weights accentuate signals from point scatterers. Our detection performance results show that $\mathrm{CF}$ has a positive weighting scheme to the DAS image at low SNR and $P_{\text {FA }}$ values.

\section{APPENDIX A}

For the speckle reduction methods in Section IV-D, we chose to use the built-in versions in MATLAB: wiener2, imnlmfilt, imdiffusefilt, imbilatfilt, and filter 2 . Table $\mathrm{V}$ presents the filter settings we used in this study. The parameter values corresponding to speckle background smoothing of $\sigma / \mu \approx 0.3$ are emphasized in bold.

Table V: Speckle Reduction Parameter Settings

\begin{tabular}{|c|c|c|}
\hline Method & Parameter Names & Parameter Values \\
\hline Wiener & Window sizes & $\begin{array}{l}5 \times 7,7 \times 9 \\
\mathbf{9} \times \mathbf{1 3}, 11 \times 15\end{array}$ \\
\hline Non-local means & $\begin{array}{l}\text { DegreeOfSmoothing }(\times \mathrm{STD}) \text {, } \\
\text { ComparisonWindowSize, } \\
\text { SearchWindowSize }\end{array}$ & $\begin{array}{l}{[1,5,11],[1,7,13]} \\
{[\mathbf{1 , 7 , 2 3}],[1,9,21]}\end{array}$ \\
\hline $\begin{array}{l}\text { Anisotropic } \\
\text { diffusion }\end{array}$ & $\begin{array}{l}\text { NumberOfIterations, } \\
\text { GradientThreshold }(\times \text { STD })\end{array}$ & $\begin{array}{l}{[7,1.5],[9,1.5]} \\
{[1 \mathbf{1 3}, 2],[20,2]}\end{array}$ \\
\hline Bilateral & $\begin{array}{l}\text { DegreeOfSmoothing }\left(\times \mathrm{STD}^{2}\right) \text {, } \\
\text { spatialSigma }\end{array}$ & $\begin{array}{l}{[1,5],[1,9]} \\
{[2,5],[3,5]}\end{array}$ \\
\hline Block averaging & Window sizes & $\begin{array}{l}5 \times 7,7 \times 9 \\
\mathbf{7} \times \mathbf{1 1}, 9 \times 13\end{array}$ \\
\hline
\end{tabular}

\section{ACKNOWLEDGMENT}

The authors would like to thank O. M. H. Rindal for discussions regarding The UltraSound Toolbox (USTB) [41].

\section{REFERENCES}

[1] M. E. Anderson, M. S. Soo, R. C. Bentley, and G. E. Trahey, "The detection of breast microcalcifications with medical ultrasound," $J$. Acoust. Soc. Am., vol. 101, no. 1, pp. 29-39, Jan. 1997.

[2] M. E. Anderson, M. S. C. Soo, and G. E. Trahey, "Microcalcifications as elastic scatterers under ultrasound," IEEE Trans. Ultrason., Ferroelectr., Freq. Control, vol. 45, no. 4, pp. 925-934, July 1998.

[3] — , "Optimizing visualization of breast microcalcifications," in Proc. IEEE Int. Ultrason. Symp. (IUS), vol. 2, Oct. 2000, pp. 1315-1320.
[4] P. Machado, J. R. Eisenbrey, M. Stanczak, B. C. Cavanaugh, L. M. Zorn, and F. Forsberg, "Ultrasound detection of microcalcifications in surgical breast specimens," Ultrasound Med. Biol., vol. 44, pp. 1286 - 1290, March 2018.

[5] E. Flørenæs, S. Solberg, J. Kvam, O. F. Myhre, O. M. Brende, and B. Angelsen, "In vitro detection of microcalcifications using dual band ultrasound," in Proc. IEEE Int. Ultrason. Symp. (IUS), Sep. 2017, pp. $1-4$.

[6] A. A. Ray, D. Ghiculete, K. T. Pace, and R. J. D. Honey, "Limitations to ultrasound in the detection and measurement of urinary tract calculi," Urology, vol. 76, no. 2, pp. 295 - 300, March 2010.

[7] J. E. Tierney, S. G. Schlunk, R. Jones, M. George, P. Karve, R. Duddu, B. C. Byram, and R. S. Hsi, "In vitro feasibility of next generation nonlinear beamforming ultrasound methods to characterize and size kidney stones," Urolithiasis, vol. 47, pp. 181-188, Jan. 2018.

[8] G. Matrone, M. A. L. Bell, E. Gonzalez, and A. Ramalli, "Shortlag spatial coherence imaging with multi-line transmission to improve needle visibility in ultrasound images," J. Acoust. Soc. Am., vol. 146, pp. 2861-2861, Oct. 2019

[9] G. Matrone, M. A. L. Bell, and A. Ramalli, "Improving the detectability of highly coherent targets in short-lag spatial coherence images with multi-line transmission," in Proc. IEEE Int. Ultrason. Symp. (IUS), Sept. 2020, pp. 1-4.

[10] K. Diamantis, A. H. Greenaway, T. Anderson, J. A. Jensen, P. A. Dalgarno, and V. Sboros, "Super-resolution axial localization of ultrasound scatter using multi-focal imaging," IEEE Trans. Biomed. Eng., vol. 65, no. 8, pp. 1840-1851, Aug. 2018.

[11] C. Oliver and S. Quegan, Understanding Synthetic Aperture Radar Images. Raleigh, NC: SciTech Publishing, 2004.

[12] D. A. Abraham, Underwater Acoustic Signal Processing: Modeling, Detection, and Estimation. Cham: Springer, 2019.

[13] S. M. Kay, Fundamentals of Statistical Signal Processing, Volume II: Detection Theory. Upper Saddle River, NJ: Prentice-Hall PTR, 1998, vol. II.

[14] — Fundamentals of Statistical Signal Processing, Volume III: Practical Algorithm Development. Pearson, 2013, vol. III.

[15] W. J. Ewens and G. R. Grant, Statistical Methods in Bioinformatics: An Introduction. New York, NY: Springer, 2005, no. II.

[16] B. C. Levy, Principles of Signal Detection and Parameter Estimation. Boston, MA: Springer, 2008.

[17] S. W. Smith, R. F. Wagner, J. M. Sandrik, and H. Lopez, "Low contrast detectability and contrast/detail analysis in medical ultrasound," IEEE Trans. Sonics Ultrason., vol. 30, no. 3, pp. 164-173, May 1983.

[18] S. W. Smith and R. F. Wagner, "Ultrasound speckle size and lesion signal to noise ratio: verification of theory," Ultrason. Imaging, vol. 6 , no. 2, pp. 174-180, April 1984.

[19] M. F. Insana and T. J. Hall, "Visual detection efficiency in ultrasonic imaging: A framework for objective assessment of image quality," $J$. Acoust. Soc. Am., vol. 95, no. 4, pp. 2081-2090, April 1994.

[20] R. J. Zemp, M. D. Parry, C. K. Abbey, and M. F. Insana, "Detection performance theory for ultrasound imaging systems," IEEE Trans. Med. Imag., vol. 24, no. 3, pp. 300-310, March 2005.

[21] A. Rodriguez-Molares, O. M. H. Rindal, J. D’hooge, S. E. Måsøy, A. Austeng, M. A. Lediju Bell, and H. Torp, "The generalized contrastto-noise ratio: A formal definition for lesion detectability," IEEE Trans. Ultrason., Ferroelectr., Freq. Control, vol. 67, no. 4, pp. 745-759, April 2020.

[22] J. C. Lacefield, W. C. Pilkington, and R. C. Waag, "Comparisons of lesion detectability in ultrasound images acquired using time-shift compensation and spatial compounding," IEEE Trans. Ultrason., Ferroelectr., Freq. Control, vol. 51, no. 12, pp. 1649-1659, Dec. 2004.

[23] J. J. Dahl, D. Hyun, M. Lediju, and G. E. Trahey, "Lesion detectability in diagnostic ultrasound with short-lag spatial coherence imaging," Ultrason. Imaging, vol. 33, no. 2, pp. 119-133, April 2011.

[24] Y. Ouyang, Z. Zhou, W. Wu, J. Tian, F. Xu, S. Wu, and P.-H. Tsui, "A review of ultrasound detection methods for breast microcalcification," Math. Biosci. Eng., vol. 16, pp. 1761-1785, March 2019.

[25] S. Huang, J. Robert, E. Radulescu, F. Vignon, and R. Erkamp, "Beamforming techniques for ultrasound microcalcification detection," in Proc. IEEE Int. Ultrason. Symp. (IUS), Sept. 2014, pp. 2193-2196.

[26] H. Torp, A. Rodriguez-Molares, and L. Løvstakken, "Optimum beamformer strategy for detecting signals in clutter noise," in Proc. IEEE Int. Ultrason. Symp. (IUS), Oct. 2015, pp. 1-4.

[27] J. A. Jensen and N. B. Svendsen, "Calculation of Pressure Fields from Arbitrarily Shaped, Apodized, and Excited Ultrasound Transducers," IEEE Trans. Ultrason., Ferroelectr., Freq. Control, vol. 39, no. 2, pp. 262-267, March 1992. 
[28] J. A. Jensen, "FIELD: A Program for Simulating Ultrasound Systems," in Proc. 10th Nordicbaltic Conf. Biomed. Imag., Suppl. 1, Part 1, vol. 4, 1996, pp. 351-353.

[29] J. A. Jensen, S. I. Nikolov, K. L. Gammelmark, and M. H. Pedersen, "Synthetic aperture ultrasound imaging," Ultrasonics, vol. 44, pp. e5 e15, December 2006, proc. of Ultrason. Int. (UI'05) and World Congress on Ultrasonics (WCU).

[30] T. L. Szabo, Diagnostic Ultrasound Imaging: Inside Out, 2nd ed. Elsevier, 2014

[31] D. H. Johnson and D. E. Dudgeon, Array signal processing: concepts and techniques. Upper Saddle River, NJ: PTR Prentice Hall, 1993, vol. XIII.

[32] R. Mallart and M. Fink, "Adaptive focusing in scattering media through sound-speed inhomogeneities: The van Cittert Zernike approach and focusing criterion," J. Acoust. Soc. Am., vol. 96, no. 6, pp. 3721-3732, Dec. 1994.

[33] J. Camacho, M. Parrilla, and C. Fritsch, "Phase Coherence Imaging," IEEE Trans. Ultrason., Ferroelectr., Freq. Control, vol. 56, no. 5, pp. 958-974, May 2009.

[34] J. Capon, "High-resolution frequency-wavenumber spectrum analysis," Proc. IEEE, vol. 57, p. 1408-1418, Aug. 1969.

[35] R. S. C. Cobbold, Foundations in Biomedical Ultrasound. New York, NY: Oxford University Press, 2007.

[36] C. B. Burckhardt, "Speckle in Ultrasound B-Mode Scans," IEEE Trans. Sonics Ultrason., vol. 25, pp. 1-6, Jan. 1978.

[37] F. J. Harris, "On the use of windows for harmonic analysis with the discrete Fourier transform," Proc. IEEE, vol. 66, pp. 51-83, Jan. 1978.

[38] D. G. Manolakis and V. K. Ingle, Applied Digital Signal Processing: Theory and Practice. Cambridge: Cambridge University Press, 2011.

[39] J. A. Hanley and B. J. McNeil, "The meaning and use of the area under a Receiver Operating Characteristic (ROC) curve," Radiology, vol. 143 , no. 1, pp. 29-36, April 1982.

[40] S. M. Hverven, O. M. H. Rindal, A. Rodriguez-Molares, and A. Austeng, "The influence of speckle statistics on contrast metrics in ultrasound imaging," in Proc. IEEE Int. Ultrason. Symp. (IUS), Sep. 2017, pp. 14.

[41] A. Rodriguez-Molares, O. M. H. Rindal, O. Bernard, A. Nair, M. A. L. Bell, H. Liebgott, A. Austeng, and L. Løvstakken, "The UltraSound ToolBox," in Proc. IEEE Int. Ultrason. Symp. (IUS), Sep. 2017, pp. $1-4$.

[42] J.-F. Synnevåg, A. Austeng, and S. Holm, "Adaptive Beamforming Applied to Medical Ultrasound Imaging," IEEE Trans. Ultrason., Ferroelectr., Freq. Control, vol. 54, no. 8, Aug. 2007.

[43] _ , "Benefits of minimum-variance beamforming in medical ultrasound imaging," IEEE Trans. Ultrason., Ferroelectr., Freq. Control, vol. 56, no. 9, pp. 1868-1879, Sep. 2009.

[44] P. C. Li and M. L. Li, "Adaptive imaging using the generalized coherence factor," IEEE Trans. Ultrason., Ferroelectr., Freq. Control, vol. 50, no. 2 pp. 128-141, Feb. 2003.

[45] J. Camacho, M. Parrilla, and C. Fritsch, "Grating-lobes reduction by application of Phase Coherence Factors," in Proc. IEEE Int. Ultrason. Symp. (IUS), Sept 2009, pp. 341-344.

[46] R. F. Wagner, S. W. Smith, J. M. Sandrik, and H. Lopez, "Statistics of speckle in ultrasound b-scans," IEEE Trans. Sonics Ultrason., vol. 30, no. 3, pp. 156-163, May 1983.

[47] O. M. H. Rindal, "Software Beamforming in Medical Ultrasound Imaging-a blessing and a curse," Ph.D. dissertation, Univ. of Oslo, Oslo, Norway, Nov. 2019, accessed on: Nov. 19, 2019.

[48] T. C. Aysal and K. E. Barner, "Rayleigh-Maximum-Likelihood Filtering for Speckle Reduction of Ultrasound Images," IEEE Trans. Med. Imag. vol. 26, no. 5, pp. 712-727, May 2007.

[49] L. H. Breivik, S. R. Snare, E. N. Steen, and A. H. S. Solberg, "RealTime Nonlocal Means-Based Despeckling," IEEE Trans. Ultrason., Ferroelectr., Freq. Control, vol. 64, no. 6, pp. 959-977, June 2007.

[50] Y. Yu and S. T. Acton, "Speckle reducing anisotropic diffusion," IEEE Trans. Image Process., vol. 11, no. 11, pp. 1260-1270, Nov. 2002.

[51] R. C. Gonzales and R. E. Woods, Digital Image Processing. Pearson, 2010, no. III.

[52] A. Buades, B. Coll, and J.-M. Morel, "A Non-Local Algorithm for Image Denoising," in Proc. IEEE Comput. Soc. Conf. Comput. Vis. Pattern Recognit., vol. 2, June 2005, pp. 60-65.

[53] P. Perona and J. Malik, "Scale-space and edge detection using anisotropic diffusion," IEEE Trans. Pattern Anal. Mach. Intell., vol. 12, no. 7, pp. 629-639, July 1990

[54] C. Tomasi and R. Manduchi, "Bilateral filtering for gray and color images," in Proc. IEEE Int. Conf. Comput. Vis., Jan. 1998, pp. 839846.

[55] S. M. Hverven, O. M. H. Rindal, A. J. Hunter, and A. Austeng, "Point scatterer enhancement in ultrasound by wavelet coefficient shrinkage," in Proc. IEEE Int. Ultrason. Symp. (IUS), Sep. 2017, pp. 1-4.

[56] D.-L. Liu and R. C. Waag, "About the Application of the Van CittertZernike Theorem in Ultrasonic Imaging," IEEE Trans. Ultrason., Ferroelectr., Freq. Control, vol. 42, no. 4, pp. 590-601, July 1995.

[57] O. M. Hoel Rindal, A. Austeng, and A. Rodriguez-Molares, "Resolution measured as separability compared to full width half maximum for adaptive beamformers," in Proc. IEEE Int. Ultrason. Symp. (IUS), Sep. 2020, pp. 1-4.

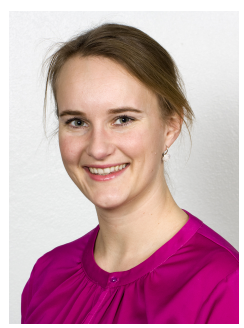

Stine Hverven Thon (S'17) was born in Oslo, Norway, in 1989. She received the M.Sc. degree in applied physics and mathematics from the Norwegian University of Science and Technology (NTNU), Trondheim, Norway, in 2013. From 2013 to 2016, she worked in DNV GL as an engineer and technical advisor on fluid dynamics and flow technology. She is currently a Ph.D. candidate in medical ultrasound with the Department of Informatics, University of Oslo, Norway. Her research interests are within medical ultrasound imaging, especially point scatterer enhancement and algorithms that improve image quality and assessment.

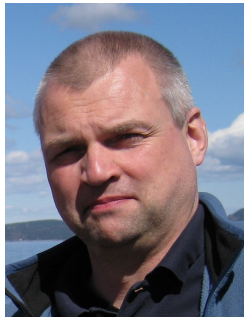

Roy Edgar Hansen (M'07-SM'16) received the M.Sc. and Ph.D. degrees in physics from the University of Tromsø, Norway, in 1992 and 1999 . respectively. From 1992 to 2000 he was with the Norwegian research company TRIAD, working on multistatic sonars, multistatic radar, SAR, and underwater communications. Since 2000 , he has been with the Norwegian Defence Research Establishment (FFI), Kjeller, Norway, working in the field of synthetic aperture sonar. He is currently Principal Scientist with FFI. He is also an Adjunct Professor in acoustic imaging with the Department of Informatics, University of Oslo, Norway. His research interests include synthetic aperture sonar and radar, ultrasound imaging, sonar signal processing, and array signal processing.

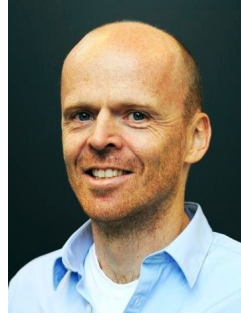

extracted from images
Andreas Austeng (S'97-M'01-SM'16) received the M.S. degree in physics and the Ph.D. degree in computer science from the University of Oslo, Norway, in 1996 and in 2001, respectively. Since 2001, he has been a Postdoctoral Research Fellow and an Associate Professor with the Department of Informatics, University of Oslo, where he is currently a Professor with the Digital Signal Processing and Image Analysis Group. His research interests include signal processing for acoustical imaging and algorithms that aim to improve the quality of information 